\title{
Alle Jahre wieder... Studentischer Parkeinsatz in Thammenhain
}

\author{
Jenny Pfriem und Nora Kindermann
}

$\mathrm{V}$

om 22. bis 25. November 2018 fand bereits zum fünften Mal - bei bestem, zeitweise sogar sonnigem Herbstwetter unser Parkseminar im Schlosspark Thammenhain statt. Die stark praxisorientierte Veranstaltung hat sich bei den Studierenden der Landschaftsarchitektur mittlerweile als feste Größe im Rahmen ihres Studiums etabliert, und so verwunderte es auch nicht, dass der Andrang groß und die freien Plätze schon vor Beginn des Wintersemesters ausgebucht waren. Auch bei der Thammenhainer Bevölkerung und den Gästen des Schlosses ist die Begeisterung für die geleisteten Arbeiten spürbar. So hat sich die im letzten Jahr in Pflege genommene Insel im Schwanenteich binnen kürzester Zeit zu einem besonderen Ort des Parkerlebnisses entwickelt und zudem die Bevölkerung zu weiteren Maßnahmen inspiriert: Die Insel mit der markanten Solitäreiche, von welcher man wunderbare Sichtbeziehungen zu Park und Schloss genießen kann, ist nun nicht allein mit dem Ruderboot zu erreichen, sondern kann auch mit einer Fähre besucht werden (Abb. 1). ${ }^{1}$ Die positive Rezeption unserer Arbeit spornt zu weiteren Taten an.

In diesem Jahr agierten drei Arbeitsgruppen im Park. Eine Gruppe beschäftigte sich mit der Grotte und deren Umfeld. Einige der hier im letzten Jahr durchgeführten Pflanzungen waren durch starke Stürme im Nachgang des letzten Seminars teilweise wieder zu Nichte gemacht worden, so dass erneuter Handlungsbedarf bestand. Neben der Pflanzung von Strauchgruppen, u.a. mit Pimpernuss (Staphylea pinnata und colchica), welche die Grotte gestalterisch besser in den
Park einbinden sollen, erfolgte die fachgerechte Pflanzung einer Hemlocktanne (Tsuga canadensis) (Abb. 2) als immergrünem Hintergrund des Bauwerks. Aufgrund der monatelang ausgebliebenen Niederschläge wies nur die oberste Bodenschicht Feuchtigkeit auf, darunter folgte völlige Trockenheit. Um dennoch ein erfolgreiches Anwachsen der Gehölze zu garantieren, waren vor der Pflanzung umfangreiche Vorbereitungsmaßnahmen notwendig (Abb. 3). Zunächst wurde der feste Boden durch gründliches Umgraben gelockert und ausgiebig gewässert. Nach der Pflanzung wurde erneut gewässert, mit Hornspänen gedüngt und die Erdschicht mit Rindenhäcksel abgedeckt, um ein schnelles Austrocknen des Bodens zu vermeiden.

Auch die Arbeiten in der Gehölzfläche zwischen den Parkferienhäusern und der Obstbaumwiese wurden weiter forciert und dem dortigen Aufwuchs, vor allem SpitzAhorn, Robinie und Brombeeren, erneut der Kampf angesagt. Besonderes Augenmerk galt diesmal den noch auf der Fläche vorhandenen Gehölzstubben und ihren Austrieben, die in mühsamer und kräftezehrender Arbeit mit Spaten, Axt und Säge entfernt wurden (Abb. 4 und 5). Den Abschluss der Arbeiten in diesem Bereich bildete die Anlage einer Strauchgruppe aus Kolbenspiere (Spiraea billardii) zur Ergänzung der vorhandenen räumlichen Fassung der Obstbaumwiese. Das hierzu benötigte Pflanzenmaterial konnte vor Ort gewonnen werden.

Einen optischen Höhepunkt des diesjährigen Seminars schuf die dritte Gruppe mit der Freistellung einer Sichtbeziehung von der Jugendstilbrücke am südwestlichen Ende 


\section{Studentischer Parkeinsatz in Thammenhain}

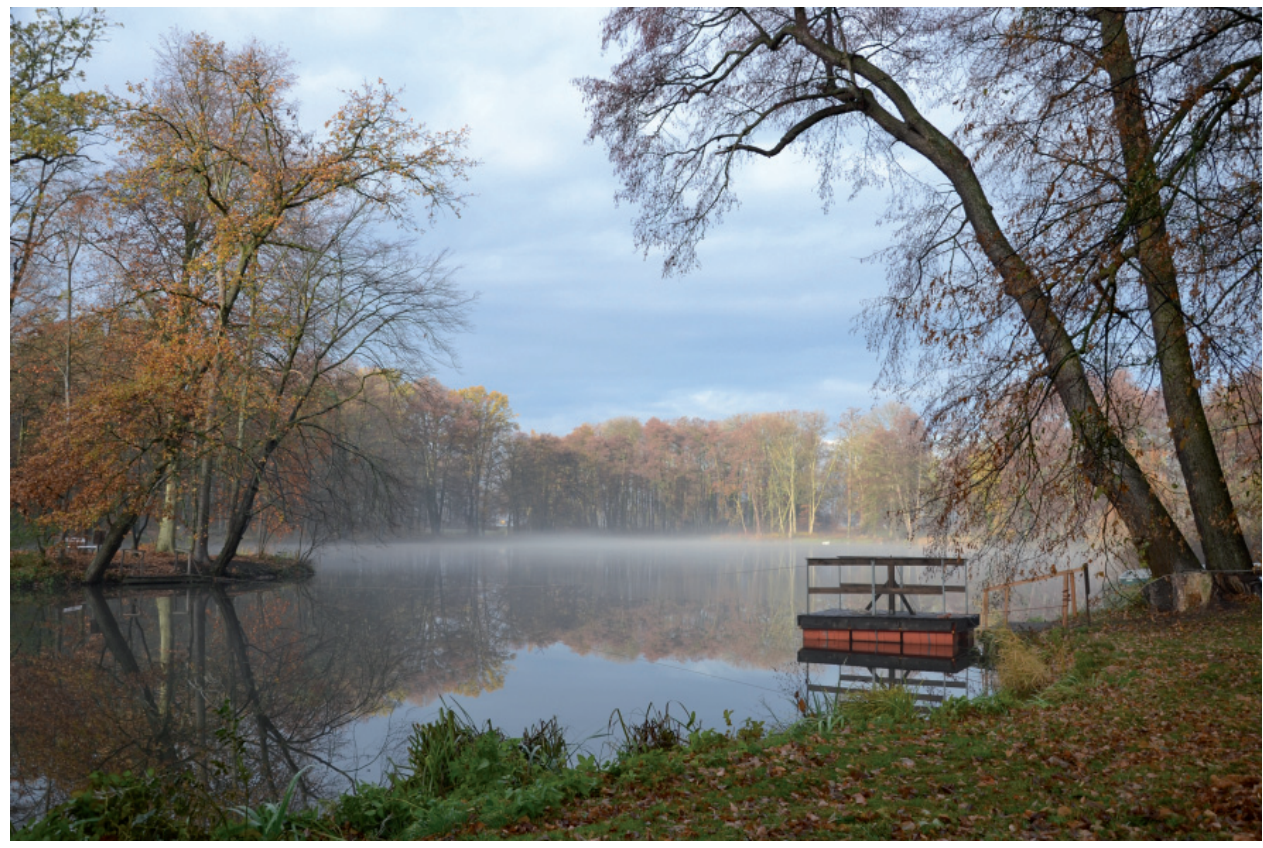

1 | Fähre zur Insel im Schwanenteich, Fotografie: Jenny Pfriem, 2018.

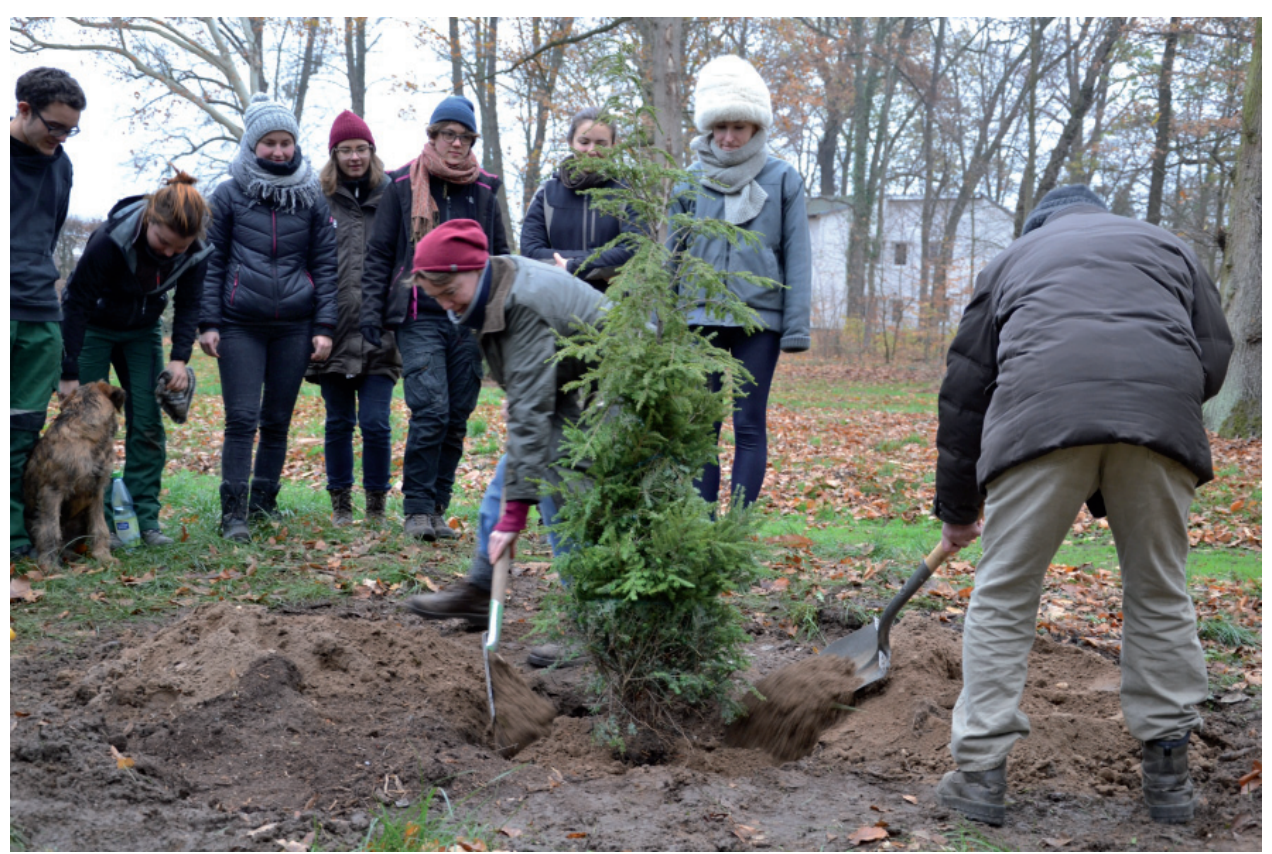

2 | Pflanzung der Hemlocktanne im Bereich der Grotte, Fotografie: Jenny Pfriem, 2018. 


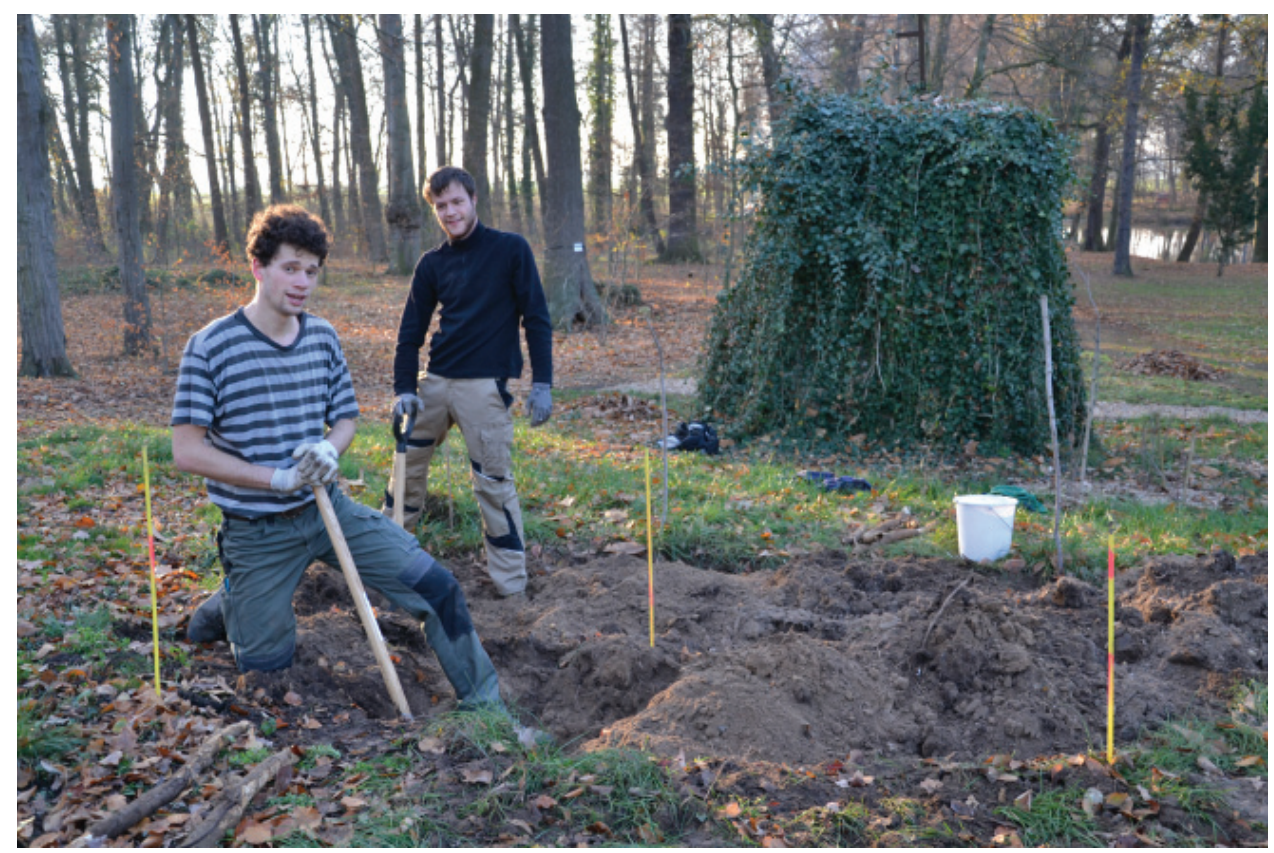

3 | Studenten beim Ausheben einer Pflanzgrube im Umfeld der Grotte, Fotografie: Jenny Pfriem, 2018.

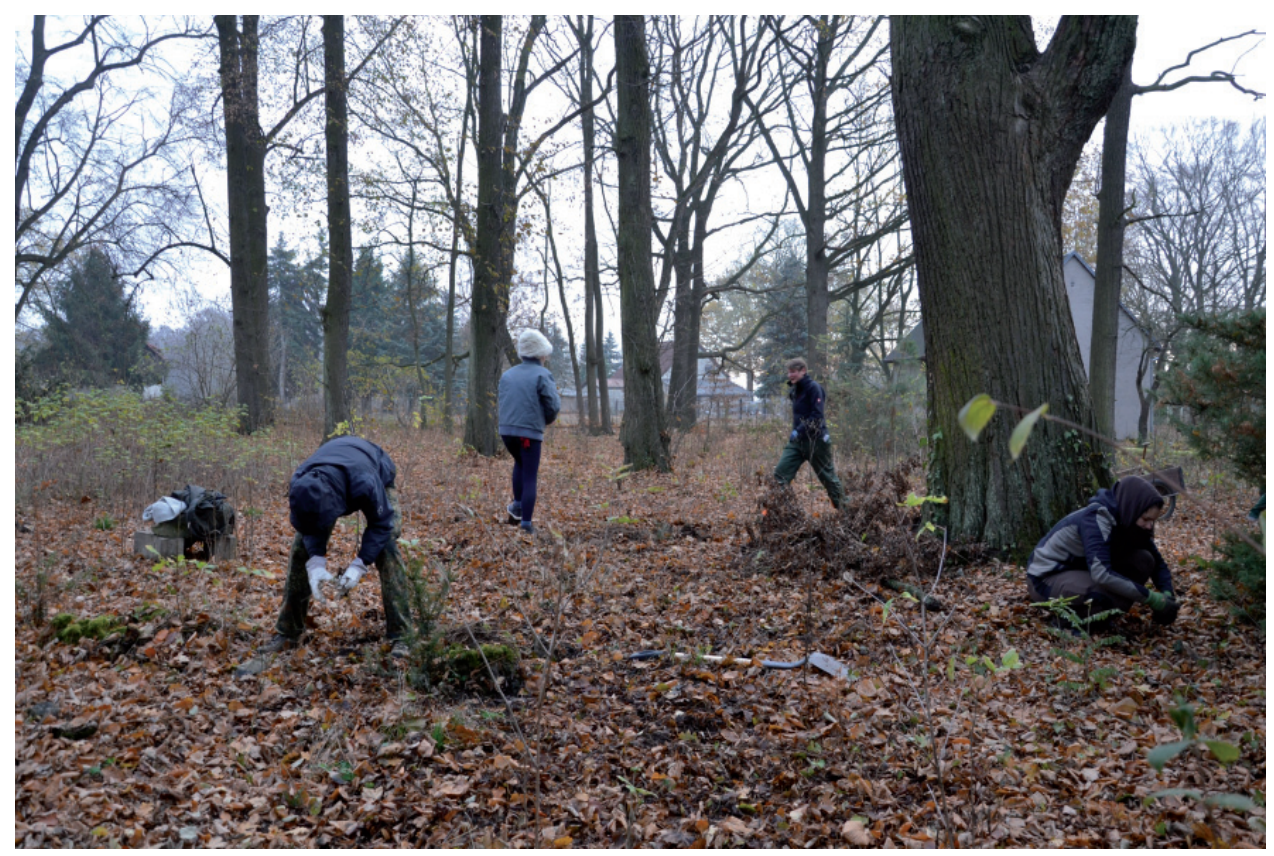

4 | Die großflächige Beseitigung von Spitz-Ahornaufwuchs in der Gehölzfläche zwischen Obstbaumwiese und Parkferienhäusern wurde weiter vorangetrieben, Fotografie: Jenny Pfriem, 2018. 


\section{Studentischer Parkeinsatz in Thammenhain}

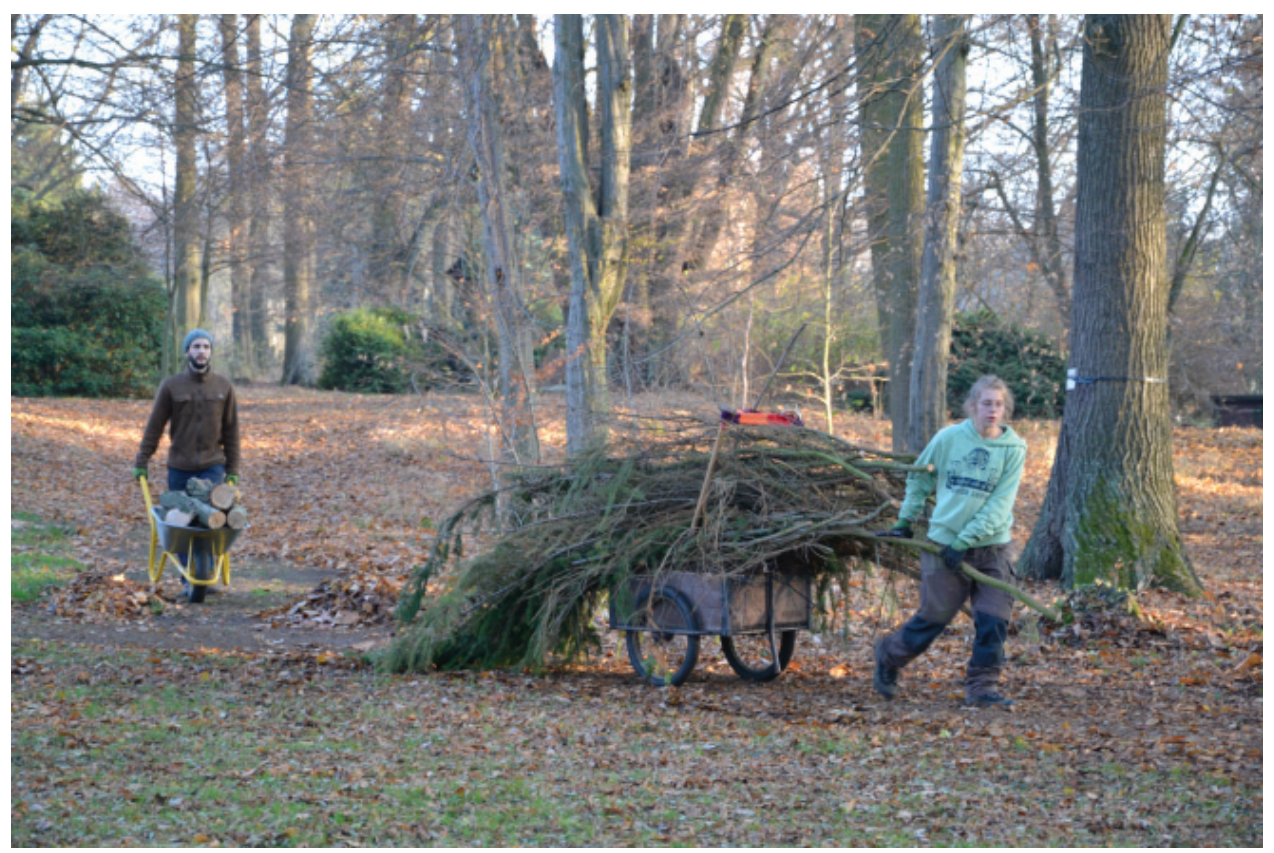

5 | Bei der Beseitigung des Schnittguts waren kreative Lösungen gefragt, Fotografie: Jenny Pfriem, 2018.

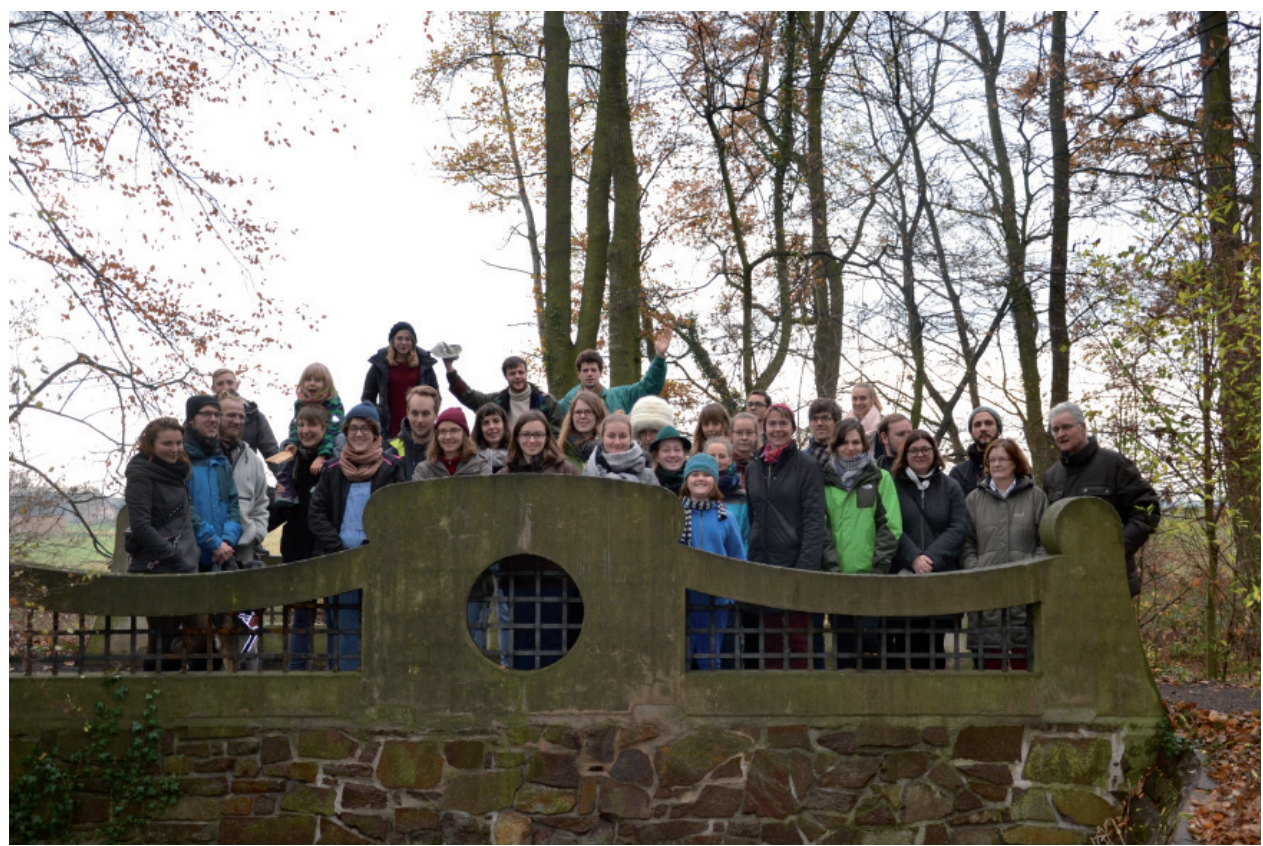

6 | Nach getaner Arbeit: Die Teilnehmer des Parkseminars an der Jugendstilbrücke am südwestlichen Ende des Schwanenteichs, im Hintergrund die wiederhergestellte Sichtbeziehung in die Landschaft, Fotografie: Nora Kindermann, 2018. 
des Schwanenteichs in Richtung Süden. Mit dem neu gewonnenen Ausblick in einen Teil der umgebenden Kulturlandschaft konnte eine besondere neue Qualität für den Park erreicht werden. Die Wiederherstellung dieser historisch belegten Landschaftsaussicht wurde durch gezielte Pflegemaßnahmen, vor allem durch die Beseitigung von Aufwuchs und einiger abgängiger Gehölze erlangt (Abb. 7 und 8). Auch der Ablauf des Schwanenteichs, der unter der Brücke hindurch entlang der Parkgrenze verläuft, wurde von Aufwuchs freigestellt. Damit ist der Bachlauf nun nicht länger nur akustisch wahrnehmbar sondern auch visuell erlebbar. Zusätzlich erfolgte im angrenzenden Bereich des Dammwegs, der ehemaligen Zufahrt zum Schloss, die Freistellung des Dammbauwerks von Aufwuchs.

Darüber hinaus war auch in diesem Jahr ein Team aus zwei Baumkletterern im Einsatz. Nach unserem Arbeitseinsatz im letzten $\mathrm{Jahr}^{2}$ hatte ein starker Wintersturm die Gehölzgruppe östlich der Grotte regelrecht hinweg gefegt. Nur einer der Altbäume, dessen Krone jedoch stark in Mitleidenschaft gezogen war, konnte stehen bleiben. In routinierter Weise wurde dieser Baum zum einen verkehrssicher und zum anderen wieder ansehnlich gemacht. Auch bei zahlreichen weiteren Altbäumen entlang der Hauptwege entfernten unsere Experten das durch die Stürme bedingte Totholz aus den Kronen.

Wir möchten zum Schluss unseren Dank an alle aussprechen, die zum erfolgreichen $\mathrm{Ge}$ lingen des Parkseminars beigetragen haben. An erster Stelle sei der Herbert-Heise-Stiftung für Gartenkunst und Landschaftskultur für die diesjährige Förderung gedankt, die erneut die reibungslose Organisation und Durchführung des Parkseminars in Thammenhain ermöglichte. Einen Dank möchten wir zudem Frau Henrike Schwarz vom Landesamt für Denkmalpflege Sachsen, Sachgebiet Gartendenkmalpflege, übermitteln, welche in gewohnt kompetenter Weise die fachliche Anleitung des Seminars übernahm. Unser Dank gilt auch allen Teilnehmern, die mit ihrem großen Engagement zum Erhalt des Schlossparkes Thammenhain beigetragen haben. Wir danken außerdem ganz herzlich unseren Gastgebern, Rüdiger und Elisabeth von Schönberg, die uns wie jedes Jahr wieder großzügig aufnahmen und deren Gastfreundschaft wir genießen durften. mann in seinem Ideenmagazin für Liebhaber von Gärten eine solche Fähre in ähnlicher Funktion abgebildet und zwar die Sternfähre im Park an der Ilm in Weimar, in: Grohmann, Johann Gottfried (Hg.): Ideenmagazin für Liebhaber von Gärten [...], Bd. 3, Heft 31, Tafel IX, Leipzig 1800.
Siehe hierzu den Bericht über das Parkseminar 2017, von Kindermann, Nora: Stürmische Zeiten in Thammenhain, in: Professur für Geschichte der Landschaftsarchitektur und Gartendenkmalpflege TU Dresden (Hg.): AHA! Miszellen zur Gartengeschichte und Gartendenkmalpflege, 4/2018, S. 132135 . 


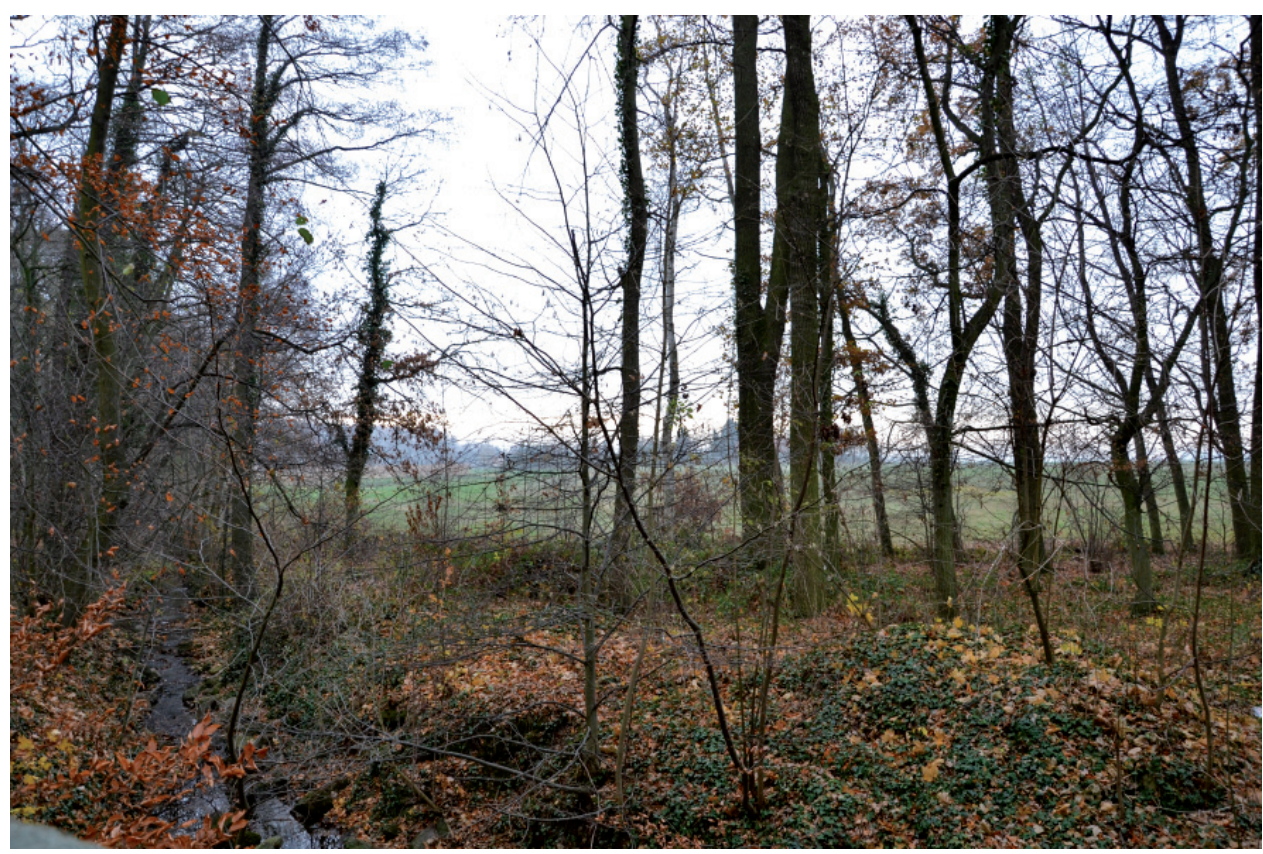

7 | Der ehemals freie Blick von der Jugendstilbrücke in Richtung Süden ist noch durch Gehölzaufwuchs verstellt, Fotografie: Jenny Pfriem, 2018.

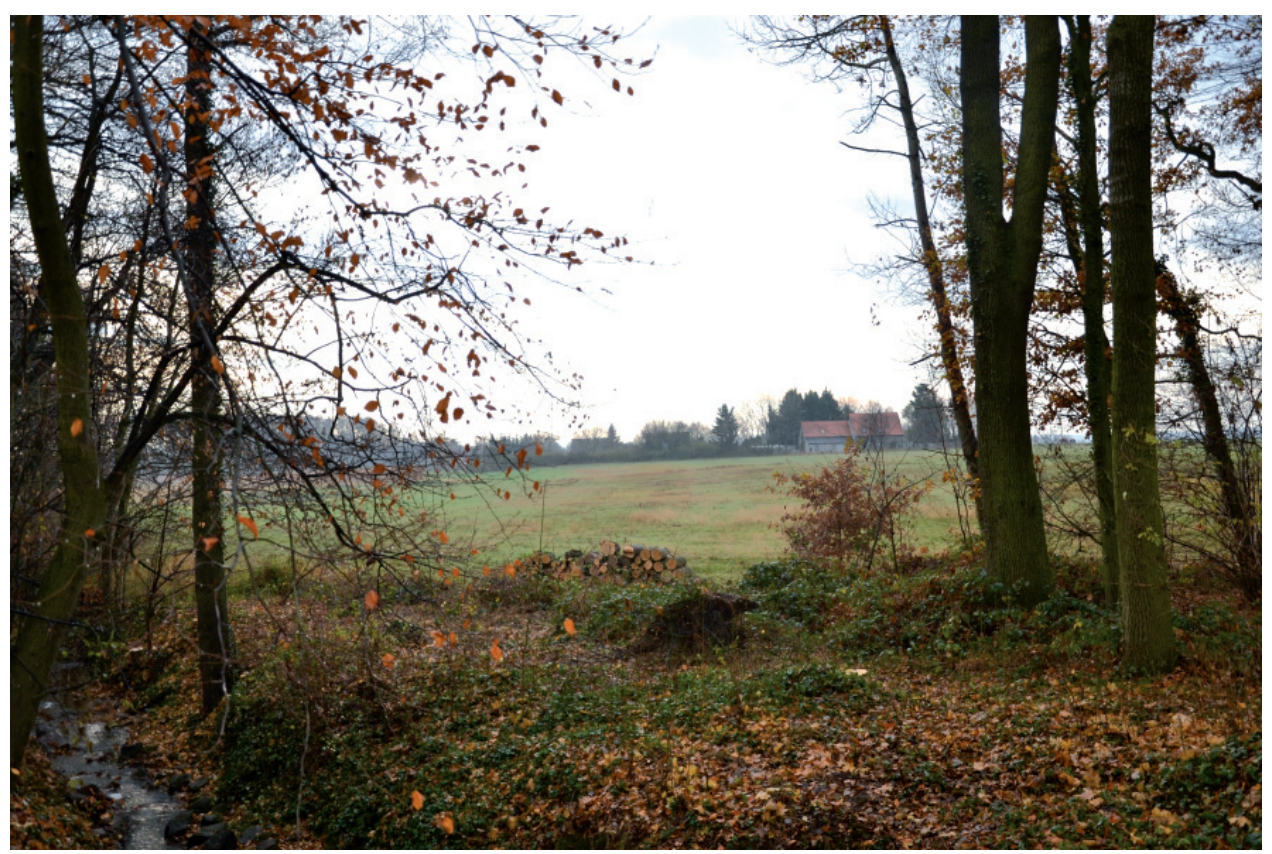

8 | Das Ergebnis kann sich sehen lassen: Freigestellte Sicht in die Kulturlandschaft nach Entfernung des Gehölzaufwuchses, Fotografie: Jenny Pfriem, 2018. 